REVIEW ARTICLE

\title{
Maternal immune activation: reporting guidelines to improve the rigor, reproducibility, and transparency of the model
}

\author{
Amanda C. Kentner ${ }^{1}$, Staci D. Bilbo ${ }^{2,3}$, Alan S. Brown ${ }^{4,5}$, Elaine Y. Hsiao ${ }^{6}$, A. Kimberley McAllister ${ }^{7}$, Urs Meyer $^{8,9}$, Brad D. Pearce $^{10}$,
} Mikhail V. Pletnikov ${ }^{11}$, Robert H. Yolken ${ }^{12}$ and Melissa D. Bauman ${ }^{13}$

The 2017 American College of Neuropychopharmacology (ACNP) conference hosted a Study Group on 4 December 2017, Establishing best practice guidelines to improve the rigor, reproducibility, and transparency of the maternal immune activation (MIA) animal model of neurodevelopmental abnormalities. The goals of this session were to (a) evaluate the current literature and establish a consensus on best practices to be implemented in MIA studies, (b) identify remaining research gaps warranting additional data collection and lend to the development of evidence-based best practice design, and (c) inform the MIA research community of these findings. During this session, there was a detailed discussion on the importance of validating immunogen doses and standardizing the general design (e.g., species, immunogenic compound used, housing) of our MIA models both within and across laboratories. The consensus of the study group was that data does not currently exist to support specific evidence-based model selection or methodological recommendations due to lack of consistency in reporting, and that this issue extends to other inflammatory models of neurodevelopmental abnormalities. This launched a call to establish a reporting checklist focusing on validation, implementation, and transparency modeled on the ARRIVE Guidelines and CONSORT (scientific reporting guidelines for animal and clinical research, respectively). Here we provide a summary of the discussions in addition to a suggested checklist of reporting guidelines needed to improve the rigor and reproducibility of this valuable translational model, which can be adapted and applied to other animal models as well.

Neuropsychopharmacology (2019) 44:245-258; https://doi.org/10.1038/s41386-018-0185-7

\section{BACKGROUND}

Brief review of epidemiologic findings of MIA

The MIA model of neuropsychiatric disorders was derived from a series of epidemiologic studies, which demonstrated associations between maternal infections during pregnancy and schizophrenia [1-3]. The most informative of these studies are based on large population-based birth cohorts employing maternal serum, neonatal blood spots, and other specimens from biobanks. Nested case-control studies in these cohorts have provided evidence supporting the MIA model. Among numerous findings, these studies have revealed associations between schizophrenia and maternal serum antibody to influenza, maternal serum/neonatal antibodies to rubella [4], Toxoplasma gondii [5, 6], and herpes simplex virus type $2[7,8]$ and cytomegalovirus [9], although not all findings have been replicated.

Cohort studies derived from these populations using electronic medical records have revealed associations between several types of maternal infections and schizophrenia [1]; generally, these studies have evaluated broad classes of infections, rather than individual microbes. Other case-control studies have demonstrated relationships between inflammatory biomarkers in maternal sera. These include a positive correlation of maternal levels of the cytokines interleukin-8 [10] and TNF-a [7], and risk of schizophrenia, an inverse correlation between the levels of anti-inflammatory Th2 cytokines and risk of schizophrenia [11], a link between elevated maternal serum CRP and schizophrenia [12] and associations between elevated levels of maternal complement components and schizophrenia [13]. Some correlations, such as the one with exposure to cytomegalovirus, are associated with genetic loci suggesting a role for gene-environmental interactions $[9,14]$.

More recently, this work has been broadened to include other neuropsychiatric and neurodevelopmental disorders. Large epidemiological studies have reported increased risk of autism spectrum disorder (ASD) associated with maternal infection during pregnancy, though results vary depending on gestational timing

\footnotetext{
${ }^{1}$ School of Arts \& Sciences, Health Psychology Program, Massachusetts College of Pharmacy and Health Sciences, Boston, MA, USA; ${ }^{2}$ Department of Pediatrics, Harvard Medical School, Boston, MA, USA; ${ }^{3}$ Lurie Center for Autism, Massachusetts General Hospital for Children, Boston, MA, USA; ${ }^{4}$ Department of Psychiatry, College of Physicians and Surgeons, Columbia University, New York, NY, USA; ${ }^{5}$ New York State Psychiatric Institute, New York, NY, USA; ${ }^{6}$ Department of Integrative Biology and Physiology, University of California, Los Angeles, USA; ${ }^{7}$ Center for Neuroscience, University of California Davis, Davis, CA, USA; ${ }^{8}$ Institute of Pharmacology and Toxicology, University of Zurich-Vetsuisse,

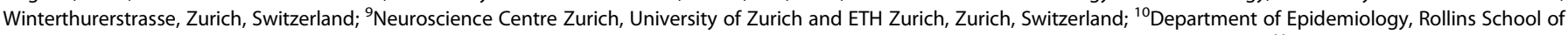
Public Health, and Graduate Division of Biological and Biomedical Sciences, Neuroscience Program, Emory University, Atlanta, GA, USA; ${ }^{11}$ Department of Psychiatry and Behavioral Sciences, Solomon H. Snyder Department of Neuroscience, Johns Hopkins University School of Medicine, Baltimore, MD, USA; ${ }^{12}$ Department of Pediatrics, Stanley

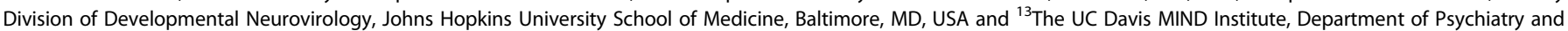
Behavioral Sciences, California National Primate Research Center, University of California, Davis, USA

Correspondence: Amanda C. Kentner (amanda.kentner@mcphs.edu) or Melissa D. Bauman (mdbauman@ucdavis.edu)
}

Received: 21 May 2018 Revised: 4 July 2018 Accepted: 2 August 2018

Published online: 21 August 2018 
of the exposure, type of infectious agent, and intensity of the maternal immune response [15-23]. Additional studies have found that maternal CRP levels are increased in pregnancies giving rise to ASD cases [24], though this finding was not replicated in a different cohort [25]. Cytokines and other soluble immune molecules, quantified in specimens such as maternal blood and amniotic fluid, have also been elevated in some pregnancies which led to ASD cases [26-29]. Maternal influenza has been associated with a fivefold increased risk of bipolar disorder [30, 31] and maternal T. gondii type I was related to affective psychoses [32].

Although there is a clear need for additional epidemiological studies, the current data suggest that, at least for a subset of women, exposure to infection during pregnancy may increase the risk of CNS disorders in offspring [1, 33]. Rather than being a risk factor for a single disease or disorder, MIA may rather be a primer for a range of psychiatric disorders [34, 35]. Moreover, the diversity of infectious agents associated with an increased risk of CNS disorders suggests that the maternal immune response may be one common link between prenatal immune challenge and altered fetal brain development. Finally, infections may be tapping into immune pathways that are shared by other environmental triggers $[36,37]$.

Overview of MIA model experimental design considerations Because the genetic, ecological, and behavioral diversity of humans is so remarkably heterogeneous, the development of MIA models in animals has been essential for testing causality, identifying molecular mechanisms, and developing new diagnostic tools and therapeutics. Indeed, rodent and, more recently, nonhuman primate (NHP) animal models of MIA have demonstrated a causal relationship between maternal infection and neuropathological and behavioral abnormalities consistent with a range of neurodevelopmental and psychiatric disorders. In these models, the immune system is activated during pregnancy using a variety of immunogens, and changes in offspring brain and behavioral development that parallel features of human disease are then measured (for reviews, see [35, 38-41]). The MIA model is emerging as a powerful translational tool to explore the effects of prenatal immune challenge on the developing fetus, though the need for a more consistent model is paramount.

Developing animal models to study complex human brain diseases, such as those associated with maternal infection, pose a major challenge to preclinical research efforts [42-44]. Historically, the validity of animal models has been determined by: (i) Construct validity-which accounts for mechanistic similarities between the model and the clinical condition, in addition to the etiological relevance of the model to human disease(s), (ii) Face validity-resemblance of outcome measures of the model to features of the human disease, and (iii) Predictive validityresponse of the model to therapeutic agents used to treat the human disease [45]. The MIA models meet all of the criteria for validity: they mimic some known biological and/or behavioral pathology and model a known disease-related risk factor, they exhibit many disease-related phenotypes, and they respond well to therapeutics used to treat CNS disorders. Although MIA models have been used most often to model ASD or SZ, the emerging consensus among leaders in the field is that prenatal infection may be relevant to a number of CNS diseases and restricting interpretation to any given human disorder may limit the utility and relevance of the MIA model $[46,47]$. Rather, prenatal immune challenge may serve as a "disease primer" into an altered trajectory of fetal brain development that, in combination with other genetic and environmental factors, may ultimately result in the emergence of ASD, SZ, or other CNS disorders [34, 35, 48].

In fact, the MIA animal models are allowing us to test the hypothesis that it is the combination of the timing of exposure, the type of immune activation (viral vs. bacterial and acute vs. chronic), environmental exposures and co-morbidities (such as stress and diet), and intensity and duration of MIA that may determine the nature of brain and behavioral alterations that manifests in offspring $[49,50]$. In spite of the challenges associated with methodological variability, described in detail below, several behavioral phenotypes associated with the model are quite reproducible (Fig. 1). Because the specific MIA induction and postnatal housing parameters appear to dictate the neuropathological and behavioral outcomes in offspring, the emerging view in our field is that we should not continue to ignore the details of how each lab generates their models but rather, we should embrace and explore those details because they may reveal critical information about the specific combination of conditions that cause risk. Moreover, we should not forget that maternal infections in humans do not always cause disease in offspring, suggesting that animal models could reveal why some pregnancies are susceptible, and others resilient, to MIA. Thus, rather than suggest specific recommendations for how MIA models should be generated, we are instead proposing that labs share specific details about their models to enhance their rigor, reproducibility, and translational relevance. Below, we describe several categories of experimental design considerations: (I) immune activation, (II) animals and their environment, and (III) outcome measures, and we propose a checklist for reporting these details. Although the focus here is on maternal models, the resulting guidelines may also be of use to postnatal immune challenge models, as this period is also clinically associated with alterations in development $[46,51,52]$.

\section{Immune activation}

Immune activation compounds: Immune molecules such as cytokines and chemokines, and the cells that produce them within the brain are critical for normal brain development. This recognition has in recent years led to the working hypothesis that inflammatory events during pregnancy, e.g., in response to infection, may disrupt the normal expression of immune molecules during critical stages of neural development and thereby contribute to the risk for neurodevelopmental disorders. The MIA hypothesis has been tested in animal models by activating the immune system during pregnancy using a variety of immunogens and then observing changes in offspring brain and behavioral development that parallel features of human CNS disorders. Although MIA can be induced by many agents [53,54], here we focus on the two most commonly used models, Poly IC and LPS.

Polyinosinic:polycytidylic acid (Poly IC) is a synthetic doublestranded RNA molecule that is commonly used as a viral mimetic. Poly IC is recognized by the pattern recognition receptor, toll-like receptor (TLR) 3, which specifically recognizes double-stranded RNA, the genetic information for many viruses [55]. Despite the fact that Poly IC is a synthetic analog, variations in its manufacture can have significant effects on its ability to drive an immune response and is emerging as an important consideration for MIA and latent inhibition $[56,57]$ models of experimental design [58]. These concerns stem, in part, from in vitro data demonstrating that Poly IC length varies among manufactures and that this variability may affect the magnitude of immune responses [59, $60]$. These results have recently been extended to in vivo studies [61] that further emphasize the importance of establishing rigorous reporting guidelines for the MIA model. Perinatal Poly IC exposure typically induces a robust febrile response, a profound increase in cytokine and chemokine production (e.g., IL-1 $\beta, \mathrm{IL}-6$, TNFa, IL-10, CXCL1) [62-64], and HPA axis activation [65-67]. However, these effects are species specific; when mice are housed below thermoneutrality (approx. $20-30^{\circ} \mathrm{C}$ ), the more robust (i.e., longer-lasting) effect of high-dose Poly IC is hypothermia [68]. Similar to behavioral symptoms seen in individuals with schizophrenia and ASD, offspring that were prenatally treated with Poly IC have been reported to have significant abnormalities in several 


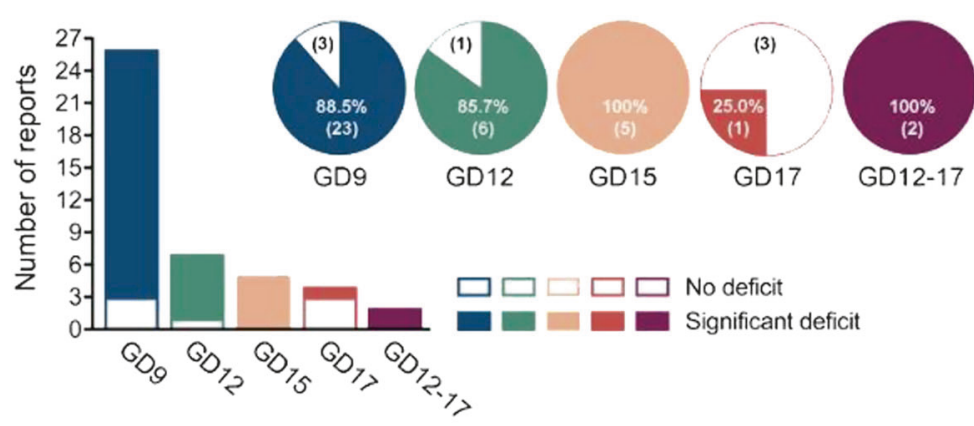

\section{b Social Approach Behavior}

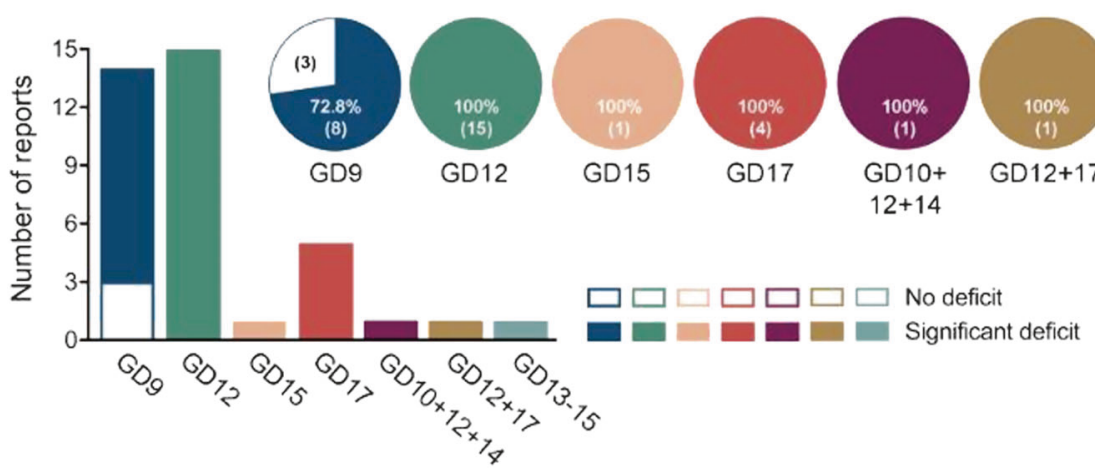

Fig. 1 Reproducibility of behavioral phenotypes that are commonly assessed in mouse models of maternal immune activation, which are based on prenatal exposure to the viral mimetic Poly IC. The bar plots show the number of studies reporting a significant deficit or no deficit in the corresponding behavioral domain after prenatal Poly IC treatment during specific gestational days (GDs). The pie charts illustrate the amount of replication (in percent) between individual studies; the numbers in brackets represent the number of studies. The corresponding studies are listed in Supplementary Table 1, which provide further information regarding basic experimental design, mouse strain, age of testing, Poly IC dosing, and sample sizes. a Reproducibility of prepulse inhibition deficits in Poly IC-based maternal immune activation models. b Reproducibility of impaired social approach behavior (sociability) in Poly IC-based maternal immune activation models.

behaviors, including deficits in the pre-pulse inhibition $[62,63$, 69-71], acoustic startle response, decreases in exploratory behavior in both open-field and novel-object tests, increased immobility in the forced swim test, and decreases in social interactions and sucrose/saccharine preferences [64, 72-82]. Importantly, many of these effects are dependent on the prenatal timing, Poly IC dosing, and genetic background of animals.

Lipopolysaccharide (LPS), the cell wall component of gramnegative bacteria, has been used to mimic infection in many animal studies because it initiates a well-characterized immune response via the activation of TLR4. The response to LPS also includes fever and is characterized by a robust increase in the expression of many cytokines and chemokines, including IL-1 $\beta$, IL6, TNFa, CXCL1, CXCL2, CXCL10, CCL2, and CCL7, among others, as well as a marked increase in circulating corticosterone [83-87]. Treatment of pregnant dams with high doses of LPS is linked to overt white matter damage, decreased oligodendrocyte development, hypo-myelination of neurons [88-90], and enhanced behavioral pain responses in adult offspring [91]. Lower doses of LPS given during the perinatal period also induce a number of long-term changes in offspring, both biochemical and behavioral, including social and cognitive deficits $[92,93]$. For each of these immunogens, the later-life impact depends on the timing of the injection [64], in addition to multiple variables we highlight below.

All available data suggest that a maternal infection can alter the developmental trajectory of the immune system and the brain. They support the idea that whereas direct neurological injury is often associated with severe neonatal infection (e.g., Borna Disease Virus or high-dose LPS), mild-to-moderate infection and subsequent cytokine exposure during key periods of brain development may result in more subtle alterations, such as altering the response or vulnerability to a subsequent insult [94]. Whether an inflammatory event within the CNS confers either resilience or vulnerability to a later challenge is a key unanswered question and most certainly depends on several factors, including timing (early, mid, or late gestation); route of administration (intraperitoneal, sub-cutaneous, intra-venous, intra-muscular, etc.); dose, source (vendor: Sigma, InvivogGen, etc.) and serotype of the immunogen; and even storage and administration conditions (time of day, room temperature, etc.) $[58,64,95,96]$. Finally, stress can significantly modify immune responses; thus, factors such as cage changes at the time of challenge should also be consistent within a study, and reported in methods. We suggest that reporting of each of these variables is critical for future research reports using these MIA models.

Immune activation validation: While it is recognized that the source, type, and administration timing of immunogens used in our inflammatory models can lead to differential behavioral and physiological outcomes, it is important to continually validate our models to ensure that discrepant findings are not due to the use of less potent or inert products. Administration of immunogens such as LPS and Poly IC activate the immune system, resulting in the release of prostaglandins and proinflammatory cytokines which have been directly tied to the induction of a series of behavioral symptoms of sickness (e.g., lethargy, reduced motivation for food and social contact, piloerection/goosebumps) [97-101], indicative of an inflammatory response. Combined, these processes drive the initiation and maintenance of fever and are part of an adaptive strategy to help organisms fight infections 
Validation of Inflammation - Microsampling vs Satellite Animal Designs

a

Experimental Immunogen Group(s) + Vehicle Group

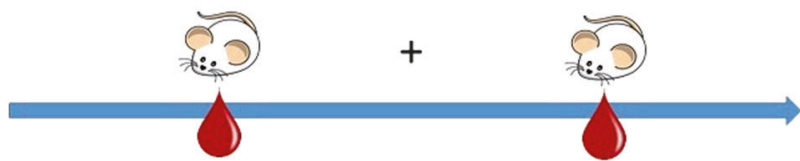

Collection of microsamples from all animal treatment groups

OR,

b Experimental Immunogen Group(s) + Vehicle Group + Satellite Animals

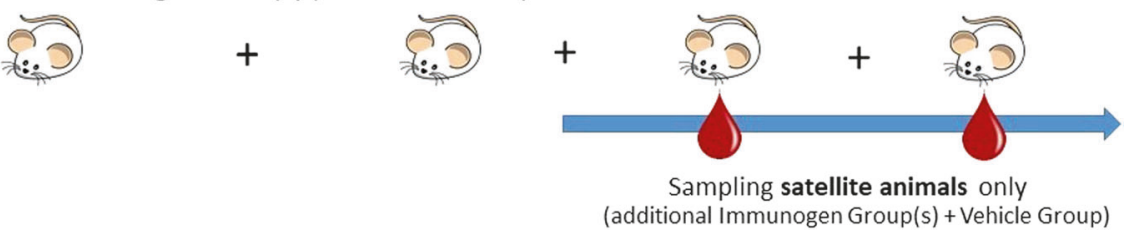

Fig. 2 Validation of inflammation in maternal immune activation (MIA) models using microsampling or satellite animal designs. a The microsampling method consists of taking small volumes of blood, plasma, and/or serum from the main experimental animals of the study in order to measure proinflammatory cytokine levels (e.g., interleukin-6) so that inflammation can be confirmed. Confounds of handling stress are an issue for this design. b In a satellite animal design, a second cohort of animals, separate from the main experimental subjects, is employed. Biological samples are collected from the satellite group only, limiting handling of the experimental group. In this design, it may be possible to miss individual differences in that some animals may not mount a measurable immune response, potentially leading to variability in the data.

[102, 103] (reviewed in [104]). The physiological and behavioral components of this "sickness response" can be quantified in order to validate commonly used animal models of inflammation.

The potency and efficacy of an immunogen can be evaluated by measuring serum or plasma levels of proinflammatory cytokines such as interleukin- $1 \beta$, interleukin- 6 , and tumor necrosis factor alpha [83, 85, 105-108]. Methodological considerations become important here-if one were to take a blood sample from every subject [109]—this introduces handling stress into the experimental protocol. Although the use of satellite animals from each treatment group can dissociate the effects of MIA from handling stress $[110,111]$ (see Fig. 2), it may be possible to miss individual differences in that some animals may not mount a measurable immune response, potentially leading to variability in the data. However, borrowing from the toxicology literature, the use of satellite groups can be useful if a sufficient number of animals for meaningful scientific interpretation are used, or if recommended guidelines are followed [109-111]. While measurement of proinflammatory cytokines can be expensive in terms of animals, reagents, and time, it may be a gold standard that labs can use to compare across their MIA models.

Another possibility to consider is the passive evaluation of cytokine-induced sickness behaviors in the home cage, the expression of which is associated with peripheral and central cytokine activation [101, 112, 113]. This option offers a noninvasive approach to confirm immune system activation without the added confound of handling stress. It is a simple, straightforward method where blinded observers can assess animals for the presence of specific sickness behaviors (e.g., ptosis, piloerection, lethargy/reduced motor activity, and huddling) across time. In one protocol, the magnitude of each specific sickness behavior is evaluated [114], whereas another recommended protocol strictly assesses the number of sickness behaviors present [115]. In both procedures, rats and mice are then assigned a total composite sickness score. These protocols have been successfully applied to male and female rodents administered LPS and Poly IC, and have been effective in pubertal, adult, and pregnant populations [115]. Similar observational methods have been developed and applied to studies using larger animals such as non-human primates [116]. Reductions in locomotor activity following immunogen challenge can be non-invasively measured in an animals' home cage using specialized recording and monitoring software to verify sickness, compared to vehicletreated control animals. For singly housed animals, specialized cages can measure food and water intake, and body weight change [117], known to be affected by immunogens as part of the inflammatory sickness response [102].

Another method for validating immunogens is to measure the inflammatory fever response. This may be done by using either implantable telemetry/data logger systems or temperature probes. A potential problem with the former option is that it requires surgery for animals that will be bred after recovery (in the case of the MIA model), which can result in high per diem expenditures. While the use of radio-frequency identification (RFID) transponders, which are implanted subcutaneously with quick recovery times, can be a reliable measure of temperature [117], the required equipment and software may be costly for laboratories with limited funds. Moreover, one-time use RFID tags can put a toll on the environment. On the other hand, reusable rectal probes or tail cuffs introduce handling, which itself can be a stressor to animals [118], eliciting hyperthermia in addition to elevated levels of hormones and cytokines [118, 119]. Less invasive infrared thermometers are improving in accuracy, but again may be cost prohibitive to some laboratories.

Another critical issue related to experimental design of MIA studies is the use of pilot experiments [120, 121]. Although it seems obvious, it may be difficult to "predict" in advance what dose, administration route, and regimen are needed for one's specific goals. Reinstating the use of dose response trials implemented in early MIA models may prove useful $[76,77,96$, $122,123]$. If, for example, one uses MIA with additional genetic or environmental manipulations, one would need to determine a dose or a range of doses that would not produce "ceiling" effects in order to be able to evaluate interactions between different factors $[73,124]$. Pilot studies could also help with consideration of what would constitute a positive control for ones' specific investigation. While negative controls usually include animals treated with vehicle and/or untreated animals, it does not seem trivial to determine what would constitute positive controls with immune stimulation. "Historical" controls based on the published and personal reports are widely used but one's specific conditions may call for additional considerations of positive controls. 
At this point, there is no consensus or guidance on how best to validate the immunogens used in our inflammatory models, or how to standardize which sources and types of immunogens we employ. Therefore, we recommend the use of our reporting chart (Table 1) so that we can collect and inform the scientific community on the most acceptable validation protocols and identify appropriate immunogens, doses, and sources.

Table 1. MIA model reporting guidelines checklist

ARRIVE Reporting Guideline \& Recommendation

Arrive MIA Model Specific Reporting Recommendation

Item Please complete this chart for each point outlined below. If not applicable, write N/A

\section{Study design}

$>$ Overview of immune activation issues

For each experiment, give brief details of the study design including:

a. The number of experimental and control groups.

b. Any steps taken to minimize the effects of subjective bias when allocating animals to treatment (e.g. randomization procedure) and when assessing results (e.g. if done, describe who was blinded and when).

c. The experimental unit (e.g. a single animal, group or cage of animals).

A time-line diagram or flow chart can be useful to illustrate how complex study designs were carried out.

\section{Experimental procedures}

$\triangleright$ Compounds

$\triangleright$ Validation measures

For each experiment and each experimental group, including controls, provide precise details of all procedures carried out. For example:

a. How (e.g. drug formulation and dose, site and route of administration, anaesthesia and analgesia used [including monitoring], surgical procedure, method of euthanasia). Provide details of any specialist equipment used, including supplier(s).

b. When (e.g. time of day).

c. Where (e.g. home cage, laboratory, water maze).

d. Why (e.g. rationale for choice of specific anaesthetic, route of administration, drug dose used).

\section{Experimental animals}

\section{$\gg$ Species/strain/vendor}

a. Provide details of the animals used, including species, strain, sex, developmental stage (e.g. mean or median age plus age range) and weight (e.g. mean or median weight plus weight range). b. Provide further relevant information such as the source of animals, international strain nomenclature, genetic modification status (e.g. knock-out or transgenic), genotype, health/immune status, drug or test naïve, previous procedures, etc.

\section{MIA Specific Reporting:}

a. General need for improved reporting in MIA model methods + reporting pilot data

- Details on pilot data:
Provide details of:

a. Compounds - source, vehicle, preparation/storage, administration route, volume administered, whether anesthetics were used at time of immune challenge.

- Name of compound:

- Catalogue number:

- Lot number:

- Vehicle control used:

- Route of administration:

- Volume administered:

- Storage conditions:

- Anesthetic (type, dose, duration) used:

b. Housing variables at injection - temperature of room at injection time, cage change at time of injection or not

- Light cycle of animal housing room:

- Time of day of injection:

- Room temperature at injection time:

- Did a cage change occur at time of injection:

c. Validation of immune activation - behavior, physiological indices and/or cytokine data, including pilot dosing data

- Method used to verify immune activation:

d. Validation of gestational timing - vaginal plug, estrous cycle, weight gain

- Method of validating gestational timing:

Additional comments:

Provide details of:

a. Species - considerations for appropriate species (mouse, rat, non human primate, other)

- Species:

b. Strain - variability in strain can influence model

-Strain:

c. Maternal/Offspring Physiological Variables at time of immune challenge - age, body weight

- Maternal Age at challenge:

- Maternal Body weight:

- Offspring Age at challenge:

- Offspring Sex:

- Offspring Body weight: 
Table 1 continued

ARRIVE Reporting Guideline \& Recommendation

Arrive MIA Model Specific Reporting Recommendation

Item Please complete this chart for each point outlined below. If not applicable, write N/A

Housing and husbandry

$\triangleright$ Cage, ventilation, bedding, enrichment

Provide details of:

a. Housing (type of facility e.g. specific pathogen free [SPF]; type of cage or housing; bedding material; number of cage companions; tank shape and material etc. for fish).

b. Husbandry conditions (e.g. breeding program, light/dark cycle, temperature, quality of water etc for fish, type of food, access to food and water, environmental enrichment).

c. Welfare-related assessments and interventions that were carried out prior to, during, or after the experiment. d. Vendor - even within the same strain, vendor can influence endpoints

- Vendor:

- Location of Vendor:

- Room/area where animals originated from:

Additional Comments:

Provide details of:

a. Caging systems

- At breeding:

Material of cage:

Cage dimensions:

- After parturition:

Material of cage:

Cage dimensions:

- At weaning:

Material of cage:

Cage dimensions:

b. Animal Holding room

- Temperature in room:

- Humidity in room:

- Ventilation system:

- Specific pathogen free [SPF]:

- Are males \& females housed in the same or separate rooms:

c. Bedding exchanges/bedding type

- Type of cage bedding used:

- Frequency of cage changes per week:

during gestation:

during neonatal period:

following weaning:

d. Breeding - bred on site or timed pregnant, how many different sires (are the same fathers breeding with both experimental and control dams)

Breeding location:

- Gestational age at shipping:

- Biological age of dams (if not listed in Section 8c):

- Number of Dams bred:

- How many times have dams been mated previously:

- How many times did the dams mate and not become pregnant:

- Are the dams primiparous or multiparous?

- What was the frequency of maternal handling during the gestational/neonatal period (e.g. cage cleanings, weighing, blood collection manipulations):

- Biological age of sires:

- Number of sires bred:

- How many times have sires been mated previously:

- How many times did the sires mate successfully (e.g. mating resulted in pregnancy, full term birth):

- If bred previously, what was the interval between mating times:

- Are sires matched to experimental and control dams:

- Describe the mating design (1:1, 1:2 etc):

e. Social enrichment - number of cage companions

- Number of cage companions prior to breeding:

- Gestational age when dam separated for parturition:

- Number of cage companions at weaning:

f. Physical enrichment - describe enrichment devices, and when enrichment is in the cage (removed when pups born? Or present throughout study), does the enrichment type change? How frequently? 
Table 1 continued

ARRIVE Reporting Guideline \& Recommendation

Arrive MIA Model Specific Reporting Recommendation

Item Please complete this chart for each point outlined below. If not applicable, write N/A

Sample size

$>$ Litter versus offspring

a. Specify the total number of animals used in each experiment, and the number of animals in each experimental group.

b. Explain how the number of animals was arrived at. Provide details of any sample size calculation used.

c. Indicate the number of independent replications of each experiment, if relevant.

\section{Allocating animals to experimental groups}

a. Give full details of how animals were allocated to experimental groups, including randomization or matching if done.

b. Describe the order in which the animals in the different experimental groups were treated and assessed.

\section{Experimental outcomes}

$\triangleright$ Behavioral testing

$>$ Physiological endpoints

Clearly define the primary and secondary experimental outcomes assessed (e.g. cell death, molecular markers, behavioral changes).

\section{Statistical methods}

a. Provide details of the statistical methods used for each analysis. b. Specify the unit of analysis for each dataset (e.g. single animal, group of animals, single neuron).

c. Describe any methods used to assess whether the data met the assumptions of the statistical approach.

\section{Other Disclosures}

- Describe what type of enrichment devices (and how many) are included in cage/housing room:

- Does enrichment type/access change across study?

- If so, when does enrichment type/access change- (e.g. enrichment removed prior to parturition and replaced in neonatal period):

-Additional Comments:

10 Provide details of:

\section{a. Maternal- $\mathrm{N}$ vs offspring $\mathrm{N}$}

-What is the total number of -dams/litters included in the study: -What is the total number of offspring per litter included the study:

b. Litter size and sex distribution

- What size was each litter maintained at:

- What age did culling take place at:

- How many males and females were maintained in each litter:

c. Cross fostering

- Did cross fostering occur:

- If so, at what age did cross fostering occur:

Additional Comments:

a. How many offspring per litter were used in each measure:

b. Randomization/Matching procedures

-What procedures were used to assign animals to groups:

c. Sex as a biological variable (behavioral and physiological outcomes)

- Were both males and females evaluated in each behavioral and physiological outcome:

Additional Comments:

a. Maternal behavior and pup interactions

- If maternal care was evaluated, were there differences following immunogen challenge (if so, please briefly describe):

b. Age(s) of offspring at behavioral testing/physiological evaluation endpoints:

c. Order of testing (e.g. behavioral test order)

- Were animals evaluated in a counter-balanced order in terms of: presentation of tests to each animal:

order of experimental/control groups run through each test: - What was the inter-test interval if a single animal underwent a battery of tests:

Additional Comments:

a. Unit of analysis for each data set

- Is the unit ( $n$ ) of each analysis based on number of litters, or number of animals used per group:
Please make note of any other extraneous variables that you would like to report (e.g. fire alarms, construction, temporary relocations, other variables that you think we should be considering in our studies etc.):

The recommended use of this reporting form is to fill it out and include it as supplemental material for each of your laboratory's research publications. If there are difficulties utilizing the fillable form (Supplementary Table 2) associated with this article please contact one of the corresponding authors to request a copy. The authors give permission for this table to be edited for use in reporting on other animal models (e.g. postnatal immune challenge models, early life stress models) as appropriate. 


\section{Animals and environment}

Species, strain, and vendor considerations: The Poly IC MIA model has attracted investigators with expertise in mouse, rat, and NHP models, which in turn has allowed the field to capitalize on the unique advantages of each species [125]. Cross-species comparisons allow preclinical researchers to compare the effects of prenatal immune challenge on evolutionarily conserved behavioral and biological outcome measures [126, 127].

Mouse models have laid the foundation for understanding the effects of MIA on fetal brain development and will undoubtedly continue to be an important species in MIA research, especially in models that incorporate genetic susceptibility. Rat models offer many of the advantages of mouse models in terms of cost, short gestational period, and the potential for genetic modifications, but also have more complex brains and display an enriched repertoire of social behavior [128]. Given that rodents comprise the vast majority of MIA model studies, it is also important to consider how strain and vendor can influence experimental outcomes. It is well established that strains of mice and rats exhibit strain-specific biological and behavioral profiles, and not unexpected that different strains may yield different outcomes in the MIA model [129]. However, mounting evidence suggests that mice from the same strain, but different vendors, may also respond differently to prenatal immune challenge due to composition of maternal gut bacteria [130]. The field of MIA research may also benefit from species that allow for a more sophisticated evaluation of social and cognitive abilities and underlying neural systems, such as the rhesus monkey [131, 132].

Although the NHP model may provide a bridge from rodent models to human disease [133], the increased costs and ethical considerations constrain the use of NHPs in research. We therefore suggest careful evaluation of which species and/or strain is most appropriate for the MIA model outcome measures of interest and reporting species, strain, and vendor details in methods. It is also important to note that species differ in maternal cytokine response, placenta function, litter size, and length of gestation. Extrapolating gestational timing across species is not always straightforward, as the gestational period of rhesus monkeys (165 days) and humans ( 280 days) is much longer than that of mice/rats (18-23 days) [134]. Moreover, the timing of implantation and development may be delayed following copulation due to the length of sperm viability and embryonic diapause $[135,136]$. For these reasons, the method of confirming gestation should also be reported (see Table 1), whether through identification of a mating plug or by estrous cycle tracking to confirm a sustained diestrus phase, for example.

Sample size, sex determination, and group allocation: Similar to all animal studies, experiments with MIA face issues with experimental design, including the determination of appropriate group size, and important decisions on statistical analyses. Most concerns are related to the lack of use of power calculations, inadequate or non-blind randomization and group allocations, and limited consideration of baseline differences between animals. It has been suggested that animal studies follow the established practices of the human clinical trials. These issues have been extensively discussed elsewhere and we refer our readers to several excellent reviews on this topic [137-139].

Randomization must always be used to make sure that each animal is allocated to an experimental group strictly by chance. Since MIA models involve two quite different sets of experimental animals, namely, pregnant and nursing dams and their offspring, random group allocation should be done for both groups. While randomly allocating pregnant or to be pregnant females to different experimental conditions, each potential condition (i.e., factor) should be taken into account, including source of animals, mating partners, housing conditions, diets, etc. It is highly desirable that pregnant dams be either single housed after establishing the pregnancy or be randomly allocated to different cages that would include both vehicle-treated and an immune inducer-treated dams. If all dams in a cage receive the same treatment, one may have to consider this cage as the experimental unit. Relatedly, littermates share similar in utero and postnatal environments which can influence offspring physiology and behavior, resulting in litter effects [140]. Therefore, each litter, as opposed to individual offspring, should be considered an experimental unit [141]. A more detailed discussion of this issue has been reviewed elsewhere [142]. Ultimately, a very careful plan should be devised in advance to deal with offspring, including randomization for cross-fostering or testing offspring after birth. As a standardization of all possible experimental approaches involving MIA model is very difficult, investigators are encouraged to provide a detailed description of the randomization and group allocation design (even if a nonstandard design was used) to enable other investigators in the field to reproduce the exact conditions of the published study.

Rearing environment: The prenatal and rearing environment includes the home cage, caging system (see below), as well as the broader environment of the housing room and animal facility. The microbiome of the mother and her offspring undoubtedly can influence behavior, but this is difficult to control. Within the home cage, one of the key influences is the role of the dam. In MIA models, the mother exerts a combination of genetic, environmental, and behavioral influences on the postnatal characteristics of her offspring. These various maternal influences are relevant to the purported downstream effects of prenatal immune stimulation on the offspring's neurobehavioral characteristics, but they are often difficult to disentangle [81, 82, 123, 143-146]. One technique used to separate these influences is "crossfostering", in which pups are removed from one dam and "transferred" to another lactating dam [147-150]. In most current behavioral studies, cross-fostering is performed within the same strain, but there is a long history of cross-fostering between strains (and even between species) to examine maternal influences on the offspring's behavior and physiology [151] (see McCarty for an excellent review [147]). In some transgenic mouse studies, interstrain cross-fostering is also used as a breeding strategy to develop or maintain a line, or to account for genetic differences in maternal behavior [152].

The advantages of cross-fostering can be considered to partially control for a number of potential confounds in the MIA model such as litter size, sex ratio, lactation, and maternal behavior [147, 153]. However, the exact procedures used for cross-fostering are often missing from publications, and fostering per se can introduce its own set of confounding factors [152, 154, 155]. To control for effects of handling and maternal-pup disruption in cross-fostering experiments, litters may be transferred between mothers within the same treatment groups (e.g., pups from an LPS-treated mother are switched to another LPS-treated mother). As fostering paradigms increase in complexity so does the cost and time commitment for a given scientific question. Despite attempts to control for the plethora of unintended effects in MIA experiments, emerging data continue to yield unexpected and intriguing findings, such as the ability of immune molecules in milk to program hippocampal development and memory in the offspring [156].

Possible influence of caging systems: Another possible confounding factor in MIA models is the precise type of animal caging system. While researchers generally report cage sizes and the number of animals that are housed per cage [123, 124], information regarding other cage parameters is mostly omitted in published articles. A number of rodent studies, however, have provided compelling evidence suggesting that the precise type of 
animal caging system can affect the animals' hygiene status, welfare, and behavior [157-162], which in turn may significantly influence the outcomes of MIA. In this regard, we have seen a continuous shift from the use of conventional caging systems such as open-top cages (OTC) or filter-top cages (FTC) to individually ventilated cage (IVC) systems [163]. Reducing the potential spreading of airborne infections and protecting the personnel from aeroallergens have been major drivers of the increasing use of IVCs in preclinical rodent research. While the possible benefits of IVCs for laboratory staff seem reasonable, it remains a matter of debate whether preclinical rodent research benefits from IVCs or not [163]. Indeed, IVCs can alter or mask neurobehavioral phenotypes that are typically seen in more conventional caging systems such as OTCs or FTCs. One illustrative example of this are the findings of Logge et al. [164], who showed that schizophreniarelated phenotypes of neuregulin 1 (Nrg1) mutant mice were no longer detectable when the animals were raised in IVCs as compared to FTCs [164].

A wide range of factors may account for the noticeable impact of the caging systems on neurobehavioral phenotypes in preclinical rodent research. For example, IVCs differ from OTCs and FTCs in regard to airflow and intra-cage oxygen $\left(\mathrm{O}_{2}\right)$, carbon dioxide $\left(\mathrm{CO}_{2}\right)$, and ammonia $\left(\mathrm{NH}_{3}\right)$ levels $[159,165]$, and they also diverge with respect to climbing opportunities [166] and intercage olfactory and acoustic cues [167]. IVCs have further been reported to impose cold stress on laboratory mice [168] and to modulate basal immune parameters [159]. All these factors may exert a significant influence on brain development and functions $[160,169,170]$, which in turn may modify the expected research outcomes and/or undermine the reproducibility of findings across different laboratories.

Acknowledging (and dealing with) the potential impact of differences in animal caging systems seems particularly relevant for MIA models, for three main reasons: first, the precise type of caging system can affect the animals' hygiene status, thereby influencing their basal immune profiles and sensitivity to infectious or immune-stimulating agents [159, 171]. Second, offspring of MIA-exposed and control mothers are raised and kept in their home cages for a substantial amount of time, typically ranging from birth to adult stages of life. Hence, the confounding effects induced by cage-specific parameters (e.g., presence or absence of climbing opportunities or inter-cage olfactory and acoustic cues in IVCs; environmental enrichment) can be expected to be maximal in such developmental models $[114,172]$. Third, the nature and/or severity of neurobehavioral and molecular changes induced by MIA are known to be dependent on pre-pubertal and post-pubertal maturational processes [78, 124, 173, 174]. External influences brought on by cage-specific factors may modify these maturational processes and may differentially shape the MIA phenotypes as the offspring mature $[114,175]$.

Thus far, systematic efforts toward investigating the potential impact of differences in laboratory caging systems are lacking in MIA models. Because of the reasons mentioned above, however, we deem such efforts highly warranted. Moreover, we believe it is important to consider and report the precise type of caging system and enrichment devices used in current and future studies of MIA to increase reproducibility across different research laboratories.

\section{Outcome measures}

Brain and behavior outcome measures: There is increasing evidence that MIA is an environmental risk factor shared across several neurological disorders, such as ASD, schizophrenia, epilepsy, depression, and neurodegenerative diseases [48, 176]. Consistent with this, in animal models, MIA alone or combined with additional environmental or genetic susceptibility, yields offspring that display subsets of various neurochemical, neurophysiological and behavioral abnormalities, as well as immunological, gastrointestinal, or metabolic disruptions [125]. This diversity in phenotypic consequences of MIA requires comprehensive and accurate reporting of experimental methods and results for outcome measures. Relatedly, the consequences of MIA challenges appear to be enduring; disruptions in brain and behavior can be transmitted transgenerationally $[81,82,92,177]$. Although beyond the scope of these particular reporting guidelines, additional reporting measures will likely need to be established in order to address the implications of these ancestral influences.

The specific experimental methods used to induce MIA should be selected to maximize the physiological relevance of the perturbation and outcomes to the disease or condition of interest. Given that there are many variations of the MIA model that each yield differences in the types, severity and onset of physiological abnormalities seen in MIA offspring, an assessment of evidence supporting face, construct and predictive validity of the specific model of MIA to disease is needed. Consideration should be given to NIMH's Research Domain Criteria (RDoC) framework for investigating mental disorders, which incorporates units of analyses from genes, molecules and cells to circuits, physiology, and behavior. Systems approaches to consider the molecular and cellular bases of neurobehavioral changes are applied to psychological constructs belonging to key behavioral domains, such as emotion, cognition, motivation, and social behavior. Numerous constructs, such as anxiety, sensory perception, cognitive control, and social communication, are relevant to the MIA model and can be used as a framework for outcome measurement and reporting.

Primary and secondary experimental outcomes should be clearly defined. In particular, procedures should indicate at which time point each measurement occurs as well as the age of animals tested. In addition, offspring age should align with the clinical endophenotypes that are being modeled. For example, with relevance to $A S D$, vocalizations or reciprocal social interactions can be assessed beginning in early postnatal ages, whereas abnormalities in sensorimotor gating seen in schizophrenia may be better examined in adolescent animals and disruptions in learning and memory that are relevant to neurodegenerative disease may be evaluated in adult and aged animals. Consistent with the importance of considering sex as a biological variable [178], whether results for each outcome assessment are sexually dimorphic should be evaluated.

Careful consideration should be given to biological and technical variables that can alter experimental outcomes. Whether each animal undergoes a battery of tests or whether there are separate cohorts that each undergo a subset of tests should be clarified. Where possible, the order of outcome testing should be justified. For example, exposures to physical and psychological stressors can profoundly alter animal behavior [179]. As such, consideration may be given to performing behavioral assessments in order of increasing severity of stress response, to minimize effects of substantial prior exposure to stress on performance in subsequent assays. In addition, whether maternal care is evaluated in the study, or whether maternal care is expected to be altered based on existing literature, should be addressed.

Importantly, statistical methods should be clearly described and justified. In particular, consideration should be given to whether the biological sample size $(\mathrm{N})$ refers to number of mothers (litters) if the primary variable is administered maternally, or if the biological sample size $(\mathrm{N})$ refers to the number of offspring tested (e.g., when testing effects of a postnatal intervention) [180]. The biological sample size of sires should also be considered as paternal effects may also contribute to the offspring phenotype [181]. Overall, dedicated efforts to promote rigorous experimental reporting will enable improved cross-comparisons of different MIA models and assessment of reproducibility across independent studies. 


\section{SUMMARY OF REPORTING GUIDELINES AND FUTURE DIRECTIONS}

Translational impact and future directions

MIA studies in pregnant animals indicate that the maternal immune response is one of the critical links between exposure to infection during pregnancy and subsequent changes in brain and behavioral development of offspring. Mice, rat, and NHP offspring born to MIA-treated dams demonstrate significant alterations in brain and behavior development, which parallel features of human neurodevelopmental disorders [34, 35, 38-41, 176]. The translation of epidemiologic findings to MIA animal models allows for a better understanding of pathogenic mechanisms, reduces the potential for bias and confounding, and allows for crossvalidation of the associations by investigating phenotypes observed in schizophrenia and other neuropsychiatric disorders. The use of animal models also allows for study of the interactions of infection with genetic factors as well as with other environmental factors such as diet, alcohol, and drug exposures, which may be important mediators of the neural consequences of maternal infection. Future epidemiologic studies aimed at "backtranslating" findings from animal models may facilitate the identification of new risk factors, mechanistic pathways, and interacting genetic mutations. Moreover, further work in both disciplines offers the potential to better understand relationships between MIA and structural and functional brain phenotypes in neuropsychiatric disorders.

The original rationale for using Poly IC in MIA models was based on the assumption that it is not so much the specific viral agent but rather a universal immune response to all types of viruses that really contributes neurodevelopmental abnormalities. Although this appealing hypothesis has never been tested directly, MIA models have significantly advanced our understanding of how Poly IC or LPS produced immune challenge in utero could increase the risk for neurodevelopmental disorders. Since the introduction of the MIA model in early 2000, there has been a significant improvement in our understanding of the mechanisms whereby various microbial infections during pregnancy could contribute to psychopathology in offspring. It is now clear that different microbial pathogens may activate dissimilar immune pathways via activating different TLR receptors. While it is plausible that various microbes could activate a limited number of immune mechanisms, the potential differences in underlying mechanisms could be significant. Thus, an alternative approach is to use inactivated microbes that would produce an agent-specific immune activation by avoiding experimental complications of working with live pathogens. Critically, the latter fact has been historically the main attractive feature of MIA model for many neurobiologists who have limited if any skills and/or lab settings to work with infectious microbes.

Reporting guidelines

Given that preclinical research is under increasing pressure to improve reproducibility $[138,182]$, the MIA model will undoubtedly benefit from renewed interest in refining experimental design standards [183]. However, MIA models are also faced with a series of unique challenges that has made it increasingly difficult to replicate outcomes, compare across studies, and establish standard protocol guidelines. The need to establish reporting guidelines is of paramount importance for the field and has lead our group to propose the following checklist (Table 1). Modeled after the ARRIVE Guidelines and CONSORT (scientific reporting guidelines for animal and clinical research, respectively), the proposed reporting checklist is a living document tailored for the MIA model, but can easily be adapted and applied to other animal models (e.g. postnatal immune challenge models, early life stress models) as well. At this moment, we are still in the initial stages of identifying key variables central to maintaining the rigor and reproducibility of data that stem from research focused on MIA.
While we include specific reporting points, we also have an "other disclosures" section for authors to provide additional information, or communicate other variables, that they deem relevant to the literature. This is a call-to-action requesting that investigators complete the 'fillable' form of Table 1 (see Supplementary Table 2 for downloadable version) and include it as part of the supplementary materials that accompany their manuscripts. Adherence to these reporting guidelines will allow for the future collation and evaluation of reported information, ultimately leading to an established consensus on the best practices to be implemented in our studies. This will also allow for the identification of remaining research gaps, leading to the evolution of evidence-based practice design and the transmission of these findings to the research community. It is our intention to solicit endorsement of this checklist from journals through its implementation as part of the manuscript submission process in order to increase the transparency of data reporting as a standard within the MIA model. With these changes, the MIA model may help us to understand which pregnancies are most vulnerable to prenatal immune challenge, which gestational time points are most sensitive, and ultimately how to safely manage the maternal immune response during pregnancy to prevent deleterious effects on fetal brain development.

\section{ACKNOWLEDGEMENTS}

The authors would like to thank the American College of Neuropsychopharmacology (ACNP) for hosting our Study Group "Establishing best practice guidelines to improve the rigor, reproducibility, and transparency of the maternal immune activation (MIA) animal model of neurodevelopmental abnormalities", which was the inspiration for this paper. We would also like to thank Ms. Allison Lau for her assistance in formatting the manuscript. The authors acknowledge financial support from CDMRP grant W81XWH-16-1-0721 (to BDP). EYH receives support from Klingenstein-Simons Foundation Fellowship in Neuroscience, Packard Fellowship in Science and Engineering. UM receives financial support from the Swiss National Science Foundation (Grant No. 310030_169544), the Foundation for Research in Science and the Humanities at the University of Zurich, and BoehringerIngelheim. MDB is supported by the UC Davis Conte Center (NIMH; P50MH106438), the UC Davis Intellectual and Developmental Disabilities Research Center (NICHD; U54HD079125). ACK receives financial support from NIMH under Award Number R15MH114035. AKM is supported by the National Institute of Neurological Disorders and Stroke (R01-NS060125-05), the National Institute of Mental Health (P50-MH106438-01), and the Simons Foundation (SFARI no. 321998). SDB receives support from the National Institute of Environmental Health Sciences (NIEHS) R01 ES025549. ASB is supported by NIEHS 1R01ES028125 and R01 ES01900404. MVP receives support from DA-041208, the Conte Center grant MH-094268, MH083728, and The Brain and Behavior Research Foundation and the Stanley Medical Research Institute. The content is solely the responsibility of the authors and does not necessarily represent the official views of any of the financial supporters.

\section{AUTHOR CONTRIBUTIONS}

The 2017 ACNP study group was organized by ACK, BDP, and MVP based on the inspiration of a previous ACNP meeting, where AKM presented data highlighting the need for increased variable transparency in the reporting of MIA data. All authors participated in the 2018 study group at ACNP and contributed to writing sections of the paper. ACK and MDB drafted the checklist and all other authors edited. Figures were made by ACK, UM, and MDB. All middle authors are listed in alphabetical order.

\section{ADDITIONAL INFORMATION}

Supplementary Information accompanies this paper at (https://doi.org/10.1038/ s41386-018-0185-7).

Competing interests: The authors declare no competing interests.

Publisher's note: Springer Nature remains neutral with regard to jurisdictional claims in published maps and institutional affiliations. 


\section{REFERENCES}

1. Brown AS, Derkits EJ. Prenatal infection and schizophrenia: a review of epidemiologic and translational studies. Am J Psychiatry. 2010;167:261-80.

2. Brown AS, et al. Serologic evidence of prenatal influenza in the etiology of schizophrenia. Arch Gen Psychiatry. 2004;61:774-80.

3. Machon RA, Mednick SA, Huttunen MO. Fetal viral infection and adult schizophrenia-empirical findings and interpretation. Neural Dev Schizophr. 1995;275:191-202.

4. Brown AS, et al. A.E. Bennett Research Award. Prenatal rubella, premorbid abnormalities, and adult schizophrenia. Biol Psychiatry. 2001;49:473-86.

5. Blomstrom A, et al. Associations between maternal infection during pregnancy, childhood infections, and the risk of subsequent psychotic disorder-a swedish cohort study of nearly 2 million individuals. Schizophr Bull. 2016;42:125-33.

6. Brown AS, et al. Maternal exposure to toxoplasmosis and risk of schizophrenia in adult offspring. Am J Psychiatry. 2005;162:767-73.

7. Buka SL, et al. Maternal infections and subsequent psychosis among offspring. Arch Gen Psychiatry. 2001;58:1032-7.

8. Mortensen PB, et al. A Danish National Birth Cohort study of maternal HSV-2 antibodies as a risk factor for schizophrenia in their offspring. Schizophr Res. 2010;122:257-63.

9. Borglum $A D$, et al. Genome-wide study of association and interaction with maternal cytomegalovirus infection suggests new schizophrenia loci. Mol Psychiatry. 2014;19:325-33.

10. Brown AS, et al. Elevated maternal interleukin-8 levels and risk of schizophrenia in adult offspring. Am J Psychiatry. 2004;161:889-95.

11. Allswede DM, et al. Elevated maternal cytokine levels at birth and risk for psychosis in adult offspring. Schizophr Res. 2016;172:41-5.

12. Canetta $S$, et al. Elevated maternal C-reactive protein and increased risk of schizophrenia in a national birth cohort. Am J Psychiatry. 2014;171:960-8.

13. Severance EG, et al. Maternal complement C1q and increased odds for psychosis in adult offspring. Schizophr Res. 2014;159:14-9.

14. Avramopoulos D, et al. Infection and inflammation in schizophrenia and bipolar disorder: a genome wide study for interactions with genetic variation. PLos ONE. 2015;10:e0116696.

15. Atladottir $\mathrm{HO}$, et al. Maternal infection requiring hospitalization during pregnancy and autism spectrum disorders. J Autism Dev Disord. 2010;40:1423-30.

16. Atladottir $\mathrm{HO}$, et al. Autism after infection, febrile episodes, and antibiotic use during pregnancy: an exploratory study. Pediatrics. 2012;130:E1447-54.

17. Zerbo $\mathrm{O}$, et al. Is maternal influenza or fever during pregnancy associated with autism or developmental delays? Results from the CHARGE (CHildhood Autism Risks from Genetics and Environment) study. J Autism Dev Disord. 2013;43:25-33.

18. Glasson EJ, et al. Perinatal factors and the development of autism-a population study. Arch Gen Psychiatry. 2004;61:618-27.

19. Langridge AT, et al. Maternal conditions and perinatal characteristics associated with autism spectrum disorder and intellectual disability. PLos ONE. 2013;8: e50963.

20. Dodds $\mathrm{L}$, et al. The role of prenatal, obstetric and neonatal factors in the development of autism. J Autism Dev Disord. 2011;41:891-902.

21. Jiang $\mathrm{HY}$, et al. Maternal infection during pregnancy and risk of autism spectrum disorders: a systematic review and meta-analysis. Brain Behav Immun. 2016;58:165-72.

22. Lee BK, et al. Maternal hospitalization with infection during pregnancy and risk of autism spectrum disorders. Brain Behav Immun. 2015:44:100-5.

23. Zerbo $O$, et al. Maternal infection during pregnancy and autism spectrum disorders. J Autism Dev Disord. 2015;45:4015-25.

24. Brown AS, et al. Elevated maternal C-reactive protein and autism in a national birth cohort. Mol Psychiatry. 2014;19:259-64.

25. Zerbo $\mathrm{O}$, et al. Maternal mid-pregnancy C-reactive protein and risk of autism spectrum disorders: the early markers for autism study. Transl Psychiatry. 2016;6: e783.

26. Goines PE, et al. Increased midgestational IFN- $\gamma$, IL-4 and IL-5 in women bearing a child with autism: a case-control study. Mol Autism. 2011;2:1.

27. Jones $\mathrm{KL}$, et al. Autism with intellectual disability is associated with increased levels of maternal cytokines and chemokines during gestation. Mol Psychiatry. 2017;22:273-9.

28. Abdallah MW, et al. Amniotic fluid chemokines and autism spectrum disorders: an exploratory study utilizing a Danish Historic Birth Cohort. Brain Behav Immun. 2012;26:170-6.

29. Abdallah MW, et al. Amniotic fluid MMP-9 and neurotrophins in autism spectrum disorders: an exploratory study. Autism Res. 2012;5:428-33.

30. Canetta SE, et al. Serological documentation of maternal influenza exposure and bipolar disorder in adult offspring. Am J Psychiatry. 2014;171:557-63.

31. Parboosing $\mathrm{R}$, et al. Gestational influenza and bipolar disorder in adult offspring. JAMA Psychiatry. 2013;70:677-85.
32. Xiao J, et al. Serological pattern consistent with infection with type I Toxoplasma gondii in mothers and risk of psychosis among adult offspring. Microbes Infect. 2009;11:1011-8.

33. Brown AS. Epidemiologic studies of exposure to prenatal infection and risk of schizophrenia and autism. Dev Neurobiol. 2012;72:1272-6.

34. Meyer U. Prenatal poly(i:C) exposure and other developmental immune activation models in rodent systems. Biol Psychiatry. 2014;75:307-15.

35. Estes ML, McAllister AK. Maternal immune activation: implications for neuropsychiatric disorders. Science. 2016;353:772-7.

36. Gilmore JH, Jarskog LF. Exposure to infection and brain development: cytokines in the pathogenesis of schizophrenia. Schizophr Res. 1997;24:365-7.

37. Pearce BD. Modeling the role of infections in the etiology of mental illness. Clin Neurosci Res. 2003;3:271-82.

38. Boksa P. Effects of prenatal infection on brain development and behavior: a review of findings from animal models. Brain Behav Immun. 2010;24:881-97.

39. Meyer U, Feldon J. Neural basis of psychosis-related behaviour in the infection model of schizophrenia. Behav Brain Res. 2009;204:322-34.

40. Patterson PH. Immune involvement in schizophrenia and autism: etiology, pathology and animal models. Behav Brain Res. 2009;204:313-21.

41. Piontkewitz Y, Arad M, Weiner I. Tracing the development of psychosis and its prevention: what can be learned from animal models. Neuropharmacology. 2012;62:1273-89.

42. Nestler EJ, Hyman SE. Animal models of neuropsychiatric disorders. Nat Neurosci. 2010;13:1161-9.

43. Patterson PH. Maternal infection and immune involvement in autism. Trends Mol Med. 2011;17:389-94.

44. Tordjman S, et al. Animal models relevant to schizophrenia and autism: validity and limitations. Behav Genet. 2007;37:61-78.

45. Willner P. The validity of animal models of depression. Psychopharmacology. 1984;83:1-16.

46. Harvey L, Boksa P. Prenatal and postnatal animal models of immune activation: relevance to a range of neurodevelopmental disorders. Dev Neurobiol. 2012;72:1335-48.

47. Meyer U, Feldon J, Dammann O. Schizophrenia and autism: both shared and disorder-specific pathogenesis via perinatal inflammation?. Pediatr Res. 2011;69:26R-33R.

48. Reisinger $\mathrm{S}$, et al. Maternal immune activation epigenetically regulates serotonin transporter levels in adult offspring hippocampus. Amino Acids. 2015;47: 1658-9.

49. Stamou $M$, et al. Neuronal connectivity as a convergent target of gene $\times$ environment interactions that confer risk for autism spectrum disorders. Neurotoxicol Teratol. 2013;36:3-16.

50. Estes ML, McAllister AK. Immune mediators in the brain and peripheral tissues in autism spectrum disorder. Nat Rev Neurosci. 2015;16:469-86.

51. Stoll BJ, et al. Neurodevelopmental and growth impairment among extremely low-birth-weight infants with neonatal infection. J Am Med Assoc. 2004;292: 2357-65.

52. Rand KM, et al. Neonatal infection and later neurodevelopmental risk in the very preterm infant. J Pediatr. 2016;170:97-104.

53. Aguilar-Valles A, Flores C, Luheshi GN. Prenatal inflammation-induced hypoferremia alters dopamine function in the adult offspring in rat: relevance for schizophrenia. PLos ONE. 2010;5:e10967.

54. Aguilar-Valles A, Luheshi GN. Alterations in cognitive function and behavioral response to amphetamine induced by prenatal inflammation are dependent on the stage of pregnancy. Psychoneuroendocrinology. 2011;36:634-48.

55. Alexopoulou $L$, et al. Recognition of double-stranded RNA and activation of NF-kappaB by Toll-like receptor 3. Nature. 2001;413:732-8.

56. Piontkewitz Y, Arad M, Weiner I. Abnormal trajectories of neurodevelopment and behavior following in utero insult in the rat. Biol Psychiatry. 2011;70:842-51.

57. Zuckerman L, Weiner I. Maternal immune activation leads to behavioral and pharmacological changes in the adult offspring. J Psychiatr Res. 2005;39: 311-23.

58. Harvey L, Boksa P. A stereological comparison of GAD67 and reelin expression in the hippocampal stratum oriens of offspring from two mouse models of maternal inflammation during pregnancy. Neuropharmacology. 2012;62: 1767-76.

59. Zhou $Y$, et al. TLR3 activation efficiency by high or low molecular mass poly I:C. Innate Immun. 2013;19:184-92.

60. Mian MF, et al. Length of dsRNA (poly I:C) drives distinct innate immune responses, depending on the cell type. J Leukoc Biol. 2013;94: 1025-36.

61. Careaga $\mathrm{M}$, et al. Variability in PolylC induced immune response: implications for preclinical maternal immune activation models. J Neuroimmunol. 2018;323: 87-93 in press. 
62. Ballendine SA, et al. Behavioral alterations in rat offspring following maternal immune activation and ELR-CXC chemokine receptor antagonism during pregnancy: Implications for neurodevelopmental psychiatric disorders. Prog Neuropsychopharmacol Biol Psychiatry. 2015;57:155-65.

63. Song $X Q$, et al. The nuclear factor-kappa $B$ inhibitor pyrrolidine dithiocarbamate reduces polyinosinic-polycytidylic acid-induced immune response in pregnant rats and the behavioral defects of their adult offspring. Behav Brain Funct. 2011;7:50.

64. Meyer $\mathrm{U}$, et al. The time of prenatal immune challenge determines the specificity of inflammation-mediated brain and behavioral pathology. J Neurosci. 2006;26:4752-62.

65. Rotondo D, et al. Pyrogenic immunomodulators increase the level of prostaglandin E2 in the blood simultaneously with the onset of fever. Eur J Pharmacol. 1988;154:145-52.

66. Milton $\mathrm{N}$, Hillhouse $\mathrm{E}$, Milton A. Activation of the hypothalamo-pituitaryadrenocortical axis in the conscious rabbit by the pyrogen polyinosinic: polycytidylic acid is dependent on corticotrophin-releasing factor-41. J Endocrinol. 1992;135:69-75.

67. Fortier $\mathrm{ME}$, et al. Maternal exposure to bacterial endotoxin during pregnancy enhances amphetamine-induced locomotion and startle responses in adult rat offspring. J Psychiatr Res. 2004;38:335-45.

68. Cunningham $C$, et al. The sickness behaviour and CNS inflammatory mediator profile induced by systemic challenge of mice with synthetic double-stranded RNA (poly I: C). Brain Behav Immun. 2007;21:490-502.

69. Vorhees CV, et al. Prenatal immune challenge in rats: altered responses to dopaminergic and glutamatergic agents, prepulse inhibition of acoustic startle, and reduced route-based learning as a function of maternal body weight gain after prenatal exposure to poly IC. Synapse. 2012;66:725-37.

70. Bates $V$, et al. Spared motivational modulation of cognitive effort in a maternal immune activation model of schizophrenia risk. Behav Neurosci. 2018;132: 66-74.

71. Howland JG, Cazakoff BN, Zhang Y. Altered object-in-place recognition memory, prepulse inhibition, and locomotor activity in the offspring of rats exposed to a viral mimetic during pregnancy. Neuroscience. 2012;201:184-98.

72. Pantelis $C$, et al. Early and late neurodevelopmental disturbances in schizophrenia and their functional consequences. Aust NZ J Psychiatry. 2003;37: 399-406.

73. Abazyan B, et al. Prenatal interaction of mutant DISC1 and immune activation produces adult psychopathology. Biol Psychiatry. 2010;68:1172-81.

74. Reisinger SN, et al. Maternal immune activation epigenetically regulates hippocampal serotonin transporter levels. Neurobiol Stress. 2016;4:34-43.

75. Straley $\mathrm{ME}$, et al. Distinct alterations in motor \& reward seeking behavior are dependent on the gestational age of exposure to LPS-induced maternal immune activation. Brain Behav Immun. 2017;63:21-34.

76. Shi LM, et al. Maternal influenza infection causes marked behavioral and pharmacological changes in the offspring. J Neurosci. 2003;23:297-302.

77. Meyer $U$, et al. Immunological stress at the maternal-foetal interface: a link between neurodevelopment and adult psychopathology. Brain Behav Immun. 2006;20:378-88.

78. Meyer $U$, et al. Relative prenatal and postnatal maternal contributions to schizophrenia-related neurochemical dysfunction after in utero immune challenge. Neuropsychopharmacology. 2008;33:441-56.

79. Hsiao EY, Patterson PH. Activation of the maternal immune system induces endocrine changes in the placenta via IL-6. Brain Behav Immun. 2011;25:604-15.

80. Gibney SM, et al. Poly I:C-induced activation of the immune response is accompanied by depression and anxiety-like behaviours, kynurenine pathway activation and reduced BDNF expression. Brain Behav Immun. 2013;28:170-81.

81. Berger $\mathrm{S}$, et al. Impact of maternal immune activation on maternal care behavior, offspring emotionality and intergenerational transmission in $\mathrm{C} 3 \mathrm{H} / \mathrm{He}$ mice. Brain Behav Immun. 2018;70:131-40.

82. Ronovsky $\mathrm{M}$, et al. Maternal immune activation transgenerationally modulates maternal care and offspring depression-like behavior. Brain Behav Immun. 2017;63:127-36.

83. Fidel PL Jr., et al. Systemic and local cytokine profiles in endotoxin-induced preterm parturition in mice. Am J Obstet Gynecol. 1994;170:1467-75.

84. Urakubo A, et al. Prenatal exposure to maternal infection alters cytokine expression in the placenta, amniotic fluid, and fetal brain. Schizophr Res. 2001;47:27-36.

85. Walker FR, et al. A profile of the immediate endocrine, metabolic and behavioural responses following a dual exposure to endotoxin in early life. Physiol Behav. 2004;83:495-504.

86. Golan HM, et al. Specific neurodevelopmental damage in mice offspring following maternal inflammation during pregnancy. Neuropharmacology. 2005;48:903-17.
87. Ortega A, Jadeja V, Zhou H. Postnatal development of lipopolysaccharideinduced inflammatory response in the brain. Inflamm Res. 2011;60:175-85.

88. Cai $Z$, et al. Cytokine induction in fetal rat brains and brain injury in neonatal rats after maternal lipopolysaccharide administration. Pediatr Res. 2000;47:64-72.

89. Fan LW, et al. Minocycline attenuates lipopolysaccharide-induced white matter injury in the neonatal rat brain. Neuroscience. 2005;133:159-68.

90. Pang Y, Cai Z, Rhodes PG. Disturbance of oligodendrocyte development, hypomyelination and white matter injury in the neonatal rat brain after intracerebral injection of lipopolysaccharide. Brain Res Dev Brain Res. 2003;140:205-14.

91. Hodyl NA, et al. Prenatal endotoxin exposure alters behavioural pain responses to lipopolysaccharide in adult offspring. Physiol Behav. 2010;100:143-7.

92. Taylor PV, et al. Sexually dimorphic effects of a prenatal immune challenge on social play and vasopressin expression in juvenile rats. Biol Sex Differ. 2012;3:15.

93. Wischhof L, et al. Prenatal LPS-exposure-a neurodevelopmental rat model of schizophrenia-differentially affects cognitive functions, myelination and parvalbumin expression in male and female offspring. Prog Neuropsychopharmacol Biol Psychiatry. 2015;57:17-30.

94. Hagberg $H$, Mallard C. Effect of inflammation on central nervous system development and vulnerability. Curr Opin Neurol. 2005;18:117-23.

95. Bilbo SD, Schwarz JM. Early-life programming of later-life brain and behavior: a critical role for the immune system. Front Behav Neurosci. 2009;3:14.

96. Fortier ME, Luheshi GN, Boksa P. Effects of prenatal infection on prepulse inhibition in the rat depend on the nature of the infectious agent and the stage of pregnancy. Behav Brain Res. 2007;181:270-7.

97. Bluthe RM, et al. Role of IL-6 in cytokine-induced sickness behavior: a study with IL-6 deficient mice. Physiol Behav. 2000;70:367-73.

98. Kelley KW, et al. Central interleukin-1 receptors as mediators of sickness. Ann N Y Acad Sci. 1997;823:234-46.

99. Kent S, et al. Mechanisms of sickness-induced decreases in food-motivated behavior. Neurosci Biobehav Rev. 1996;20:171-5.

100. Bluthe RM, et al. Role of interleukin-1 beta and tumour necrosis factor-alpha in lipopolysaccharide-induced sickness behaviour: a study with interleukin-1 type I receptor-deficient mice. Eur J Neurosci. 2000;12:4447-56.

101. Dantzer $R$, et al. From inflammation to sickness and depression: when the immune system subjugates the brain. Nat Rev Neurosci. 2008;9:46-56.

102. Hart BL. Biological basis of the behavior of sick animals. Neurosci Biobehav Rev. 1988;12:123-37.

103. Dantzer R. Cytokine-induced sickness behavior: mechanisms and implications. Ann N Y Acad Sci. 2001;933:222-34.

104. Dantzer R, Kelley KW. Twenty years of research on cytokine-induced sickness behavior. Brain Behav Immun. 2007;21:153-60.

105. Ashdown $\mathrm{H}$, et al. The role of cytokines in mediating effects of prenatal infection on the fetus: implications for schizophrenia. Mol Psychiatry. 2006;11:47-55.

106. Blatteis $\mathrm{CM}$, et al. Signaling the brain in systemic inflammation: the role of complement. Front Biosci. 2004;9:915-31.

107. Dinarello CA. Cytokines as endogenous pyrogens. J Infect Dis. 1999;179:S294-304.

108. Steiner AA, et al. Cellular and molecular bases of the initiation of fever. PLoS Biol. 2006;4:e284.

109. Sparrow SS, et al. Opportunities to minimise animal use in pharmaceutical regulatory general toxicology: a cross-company review. Regul Toxicol Pharmacol. 2011;61:222-9.

110. Guideline IHT. Note for guidance on toxicokinetics: the assessment of systemic exposure in toxicity studies S3A. Geneva: ICH Harmonisation for Better Health; 1994.

111. Campbell $B$, Ings $B$. Toxicokinetics: a guidance for assessing systemic exposure in toxicology studies, where are we now; an S3A/S3B update (1995-2011). In: Jan Willem van der Laan, Joseph J. DeGeorge (ed.) Global approach in safety testing. Springer; Switzerland, 2013. pp. 119-58.

112. Dantzer R. Cytokine, sickness behavior, and depression. Immunol Allergy Clin North Am. 2009;29:247-64

113. Konsman JP, Parnet $P$, Dantzer R. Cytokine-induced sickness behaviour: mechanisms and implications. Trends Neurosci. 2002;25:154-9.

114. Connors EJ, et al. Environmental enrichment mitigates the sex-specific effects of gestational inflammation on social engagement and the hypothalamic pituitary adrenal axis-feedback system. Brain Behav Immun. 2014;42:178-90.

115. Kolmogorova $D$, Murray $E$, Ismail N. Monitoring pathogen-induced sickness in mice and rats. Curr Protoc Mouse Biol. 2017;7:65-76.

116. Bauman MD, et al. Activation of the maternal immune system during pregnancy alters behavioral development of rhesus monkey offspring. Biol Psychiatry. 2014;75:332-41.

117. Redfern WS, et al. Automated recording of home cage activity and temperature of individual rats housed in social groups: the Rodent Big Brother project. PLoS ONE. 2017;12:e0181068. 
118. Veening JG, et al. Stress-induced hyperthermia in the mouse: c-fos expression, corticosterone and temperature changes. Prog Neuropsychopharmacol Biol Psychiatry. 2004;28:699-707.

119. Hale KD, et al. Cytokine and hormone profiles in mice subjected to handling combined with rectal temperature measurement stress and handling only stress. Life Sci. 2003;72:1495-508.

120. Johnson PD, Besselsen DG. Practical aspects of experimental design in animal research. ILAR J. 2002;43:202-6.

121. Lancaster GA, Dodd S, Williamson PR. Design and analysis of pilot studies: recommendations for good practice. J Eval Clin Pract. 2004;10: 307-12.

122. Missault $\mathrm{S}$, et al. The risk for behavioural deficits is determined by the maternal immune response to prenatal immune challenge in a neurodevelopmental model. Brain Behav Immun. 2014;42:138-46.

123. Meyer $\mathrm{U}$, et al. Towards an immuno-precipitated neurodevelopmental animal model of schizophrenia. Neurosci Biobehav Rev. 2005;29:913-47.

124. Giovanoli $\mathrm{S}$, et al. Stress in puberty unmasks latent neuropathological consequences of prenatal immune activation in mice. Science. 2013;339:1095-9.

125. Careaga M, Murai T, Bauman MD. Maternal immune activation and autism spectrum disorder: from rodents to nonhuman and human primates. Biol Psychiatry. 2017;81:391-401.

126. Stewart AM, Kalueff AV. Developing better and more valid animal models of brain disorders. Behav Brain Res. 2015;276:28-31.

127. Belzung $C$, Lemoine $M$. Criteria of validity for animal models of psychiatric disorders: focus on anxiety disorders and depression. Biol Mood Anxiety Disord. 2011;1:9.

128. Vanderschuren LJ, Trezza V. What the laboratory rat has taught us about social play behavior: role in behavioral development and neural mechanisms. Curr Top Behav Neurosci. 2014;16:189-212.

129. Schwartzer JJ, Koenig CM, Berman RF. Using mouse models of autism spectrum disorders to study the neurotoxicology of gene-environment interactions. Neurotoxicol Teratol. 2013;36:17-35.

130. Kim S, et al. Maternal gut bacteria promote neurodevelopmental abnormalities in mouse offspring. Nature. 2017;549:528-32.

131. Chang SW, Platt ML. Oxytocin and social cognition in rhesus macaques: implications for understanding and treating human psychopathology. Brain Res. 2014;1580:57-68.

132. Platt ML, Seyfarth RM, Cheney DL. Adaptations for social cognition in the primate brain. Philos Trans R Soc Lond B Biol Sci. 2016;371:20150096.

133. Watson $D J$, et al. Blockade of dopamine $D(3)$ but not $D(2)$ receptors reverses the novel object discrimination impairment produced by post-weaning social isolation: implications for schizophrenia and its treatment. Int J Neuropsychopharmacol. 2012;15:471-84.

134. Clancy B, Darlington RB, Finlay BL. Translating developmental time across mammalian species. Neuroscience. 2001;105:7-17.

135. Birkhead TR, Moller AP. Sexual selection and the temporal separation of reproductive events - sperm storage data from reptiles, birds and mammals. Biol J Linn Soc. 1993;50:295-311.

136. Yochem DE. Spermatozoon life in the female reproductive tract of the guinea pig and rat. Biol Bull. 1929;56:274-97.

137. Festing MF, Altman DG. Guidelines for the design and statistical analysis of experiments using laboratory animals. ILAR J. 2002;43:244-58.

138. Landis SC, et al. A call for transparent reporting to optimize the predictive value of preclinical research. Nature. 2012;490:187-91.

139. Hirst JA, et al. The need for randomization in animal trials: an overview of systematic reviews. PLoS ONE. 2014;9:e98856.

140. Zorrilla EP. Multiparous species present problems (and possibilities) to developmentalists. Dev Psychobiol. 1997;30:141-50.

141. Lazic SE, Essioux L. Improving basic and translational science by accounting for litter-to-litter variation in animal models. BMC Neurosci. 2013:14:37.

142. Spencer SJ, Meyer U. Perinatal programming by inflammation. Brain Behav Immun. 2017;63:1-7.

143. Meyer $U$, et al. Prenatal and postnatal maternal contributions in the infection model of schizophrenia. Exp Brain Res. 2006;173:243-57.

144. Richetto J, et al. Prenatal versus postnatal maternal factors in the development of infection-induced working memory impairments in mice. Brain Behav Immun. 2013;33:190-200.

145. O'Leary $C_{\text {, }}$ et al. Phenotypic effects of maternal immune activation and early postnatal milieu in mice mutant for the schizophrenia risk gene neuregulin-1. Neuroscience. 2014;277:294-305.

146. Walker AK, et al. Transgenerational transmission of anxiety induced by neonatal exposure to lipopolysaccharide: implications for male and female germ lines. Psychoneuroendocrinology. 2012;37:1320-35.
147. McCarty R. Cross-fostering: elucidating the effects of genexenvironment interactions on phenotypic development. Neurosci Biobehav Rev. 2017;73: 219-54.

148. Meaney MJ, Szyf M. Environmental programming of stress responses through DNA methylation: life at the interface between a dynamic environment and a fixed genome. Dialog Clin Neurosci. 2005;7:103-23.

149. Gomez-Serrano $M$, et al. Effects of cross fostering on open-field behavior, acoustic startle, lipopolysaccharide-induced corticosterone release, and body weight in Lewis and Fischer rats. Behav Genet. 2001;31:427-36.

150. Huot RL, et al. Foster litters prevent hypothalamic-pituitary-adrenal axis sensitization mediated by neonatal maternal separation. Psychoneuroendocrinology. 2004;29:279-89.

151. Reading AJ. Effect of maternal environment on the behavior of inbred mice. J Comp Physiol Psychol. 1966;62:437.

152. Lerch $\mathrm{S}$, et al. What makes a good mother? Implication of inter-, and intrastrain strain "cross fostering" for emotional changes in mouse offspring. Behav Brain Res. 2014;274:270-81.

153. Bartolomucci $A$, et al. Cross fostering in mice: behavioral and physiological carryover effects in adulthood. Genes Brain Behav. 2004;3:115-22.

154. Caporali $P$, et al. Interaction does count: a cross-fostering study on transgenerational effects of pre-reproductive maternal enrichment. Front Behav Neurosci. 2015;9:320.

155. Roguski EE, et al. Fostering itself increases nicotine self-administration in young adult male rats. Psychopharmacology. 2013;229:227-34.

156. Liu B, et al. Maternal hematopoietic TNF, via milk chemokines, programs hippocampal development and memory. Nat Neurosci. 2014;17:97-105.

157. Bohr UR, et al. Prevalence and spread of enterohepatic Helicobacter species in mice reared in a specific-pathogen-free animal facility. J Clin Microbiol. 2006;44: 738-42.

158. Mineur YS, Crusio WE. Behavioral effects of ventilated micro-environment housing in three inbred mouse strains. Physiol Behav. 2009;97:334-40.

159. York JM, et al. Individually ventilated cages cause chronic low-grade hypoxia impacting mice hematologically and behaviorally. Brain Behav Immun 2012;26:951-8.

160. Logge W, Kingham J, Karl T. Behavioural consequences of IVC cages on male and female C57BL/6J mice. Neuroscience. 2013;237:285-93.

161. Burman O, et al. The effect of two different Individually Ventilated Cage systems on anxiety-related behaviour and welfare in two strains of laboratory mouse. Physiol Behav. 2014;124:92-9.

162. Polissidis A, et al. Assessing the exploratory and anxiety-related behaviors of mice. Do different caging systems affect the outcome of behavioral tests? Physiol Behav. 2017;177:68-73.

163. Baumans V, et al. Individually ventilated cages: beneficial for mice and men? Contemp Top Lab Anim Sci. 2002;41:13-9.

164. Logge W, Kingham J, Karl T. Do individually ventilated cage systems generate a problem for genetic mouse model research?. Genes Brain Behav. 2014;13:713-20.

165. Krohn TC, Hansen AK. Carbon dioxide concentrations in unventilated IVC cages. Lab Anim. 2002;36:209-12.

166. Kallnik M, et al. Impact of IVC housing on emotionality and fear learning in male C3HeB/FeJ and C57BL/6J mice. Mamm Genome. 2007;18:173-86.

167. Hawkins $P$, et al. Individually ventilated cages and rodent welfare: report of the 2002 RSPCA/UFAW rodent welfare group meeting. Anim Technol Welf 2003;2:23-34.

168. David JM, et al. Individually ventilated cages impose cold stress on laboratory mice: a source of systemic experimental variability. J Am Assoc Lab Anim Sci. 2013;52:738-44

169. Crabbe JC, Wahlsten D, Dudek BC. Genetics of mouse behavior: interactions with laboratory environment. Science. 1999;284:1670-2.

170. Kostomitsopoulos $\mathrm{N}$, et al. The effects of different types of individually ventilated caging systems on growing male mice. Lab Anim. 2012;41:192-7.

171. Booth JL, et al. Housing conditions modulate the severity of Mycoplasma pulmonis infection in mice deficient in class A scavenger receptor. Comp Med. 2014;64:424-39.

172. Connors EJ, et al. Environmental enrichment models a naturalistic form of maternal separation and shapes the anxiety response patterns of offspring. Psychoneuroendocrinology. 2015;52:153-67.

173. Zuckerman $L$, et al. Immune activation during pregnancy in rats leads to a postpubertal emergence of disrupted latent inhibition, dopaminergic hyperfunction, and altered limbic morphology in the offspring: a novel neurodevelopmental model of schizophrenia. Neuropsychopharmacology. 2003;28:1778-89.

174. Richetto J, et al. Prenatal immune activation induces maturation-dependent alterations in the prefrontal GABAergic transcriptome. Schizophr Bull. 2014;40:351-61. 
Maternal immune activation: reporting guidelines to improve the rigor $\ldots .$.

AC Kentner et al.

258

175. Kentner AC, et al. Environmental enrichment rescues the effects of early life inflammation on markers of synaptic transmission and plasticity. Brain Behav Immun. 2016;57:151-60.

176. Knuesel I, et al. Maternal immune activation and abnormal brain development across CNS disorders. Nat Rev Neurol. 2014;10:643-60.

177. Weber-Stadlbauer $U$, et al. Transgenerational transmission and modification of pathological traits induced by prenatal immune activation. Mol Psychiatry. 2017;22:102-12.

178. Bale TL, Epperson CN. Sex as a biological variable: who, what, when, why, and how. Neuropsychopharmacology. 2017;42:386-96.
179. Bailey KR, Crawley JN. Anxiety-related behaviors in mice. In: Buccafusco JJ, editor. Methods of behavior analysis in neuroscience. Boca Raton, FL: CRC Press/ Taylor \& Francis; 2009.

180. Lazic SE. Comment on "Stress in puberty unmasks latent neuropathological consequences of prenatal immune activation in mice". Science. 2013;340:811.

181. Mashoodh $\mathrm{R}$, et al. Maternal modulation of paternal effects on offspring development. Proc Biol Sci. 2018;285:20180118.

182. Collins FS, Tabak LA. Policy: NIH plans to enhance reproducibility. Nature. 2014;505:612-3.

183. Kilkenny C, et al. Improving bioscience research reporting: the ARRIVE guidelines for reporting animal research. J Pharmacol Pharmacother. 2010;1:94-9. 